\title{
Brain Activation During Passive and Volitional Pedaling After Stroke
}

\author{
Brice T. Cleland and Sheila Schindler-Ivens
}

Marquette University

\begin{abstract}
Background: Prior work indicates that pedaling-related brain activation is lower in people with stroke than in controls. We asked whether this observation could be explained by between-group differences in volitional motor commands and pedaling performance. Methods: Individuals with and without stroke performed passive and volitional pedaling while brain activation was recorded with functional magnetic resonance imaging. The passive condition eliminated motor commands to pedal and minimized between-group differences in pedaling performance. Volume, intensity, and laterality of brain activation were compared across conditions and groups. Results: There were no significant effects of condition and no Group $\times$ Condition interactions for any measure of brain activation. Only 53\% of subjects could minimize muscle activity for passive pedaling. Conclusions: Altered motor commands and pedaling performance are unlikely to account for reduced pedaling-related brain activation poststroke. Instead, this phenomenon may be due to functional or structural brain changes. Passive pedaling can be difficult to achieve and may require inhibition of excitatory descending drive.
\end{abstract}

Keywords: electromyography, fMRI, hemiparesis, locomotion, neuroplasticity, rehabilitation

There are many examples of altered movement-related brain activation in individuals with poststroke hemiparesis. Changes in the extent, intensity, and location of brain activation have been observed during upper and lower limb movements, in acute and chronic stroke survivors, and before and after rehabilitation (Bosnell et al., 2011; Calautti, Leroy, Guincestre, \& Baron, 2001; Cramer

(C) 2019 The Authors. Published by Human Kinetics, Inc. This is an Open Access article distributed under the terms of the Creative Commons Attribution License CC BY-NC 4.0, which permits unrestricted noncommercial use, distribution, and reproduction in any medium, provided the original work is properly cited, the new use includes a link to the license, and any changes are indicated. See http://creativecommons.org/licenses/by-nc/4.0. This license does not cover any third-party material that may appear with permission in the article. For commercial use, permission should be requested from Human Kinetics, Inc., through the Copyright Clearance Center (http://www.copyright.com).

Cleland and Schindler-Ivens are with the Clinical \& Translational Rehabilitation Health Science Program, Dept. of Physical Therapy, Marquette University, Milwaukee, WI. Address author correspondence to Brice T. Cleland at brice.cleland@gmail.com. 
et al., 1997; Dobkin, Firestine, West, Saremi, \& Woods, 2004; Enzinger et al., 2008; Kim et al., 2006; Miyai et al., 2002, 2003; Ward, Brown, Thompson, \& Frackowiak, 2003a, 2003b; Weiller, Chollet, Friston, Wise, \& Frackowiak, 1992). Such changes in brain activation are often attributed to stroke-related neuroplastic phenomena (for review, see Calautti \& Baron, 2003). This interpretation of human neuroimaging data is supported by observations from nonhuman animal studies. Experimentally induced brain lesions in rats and mice alter the excitability of remaining neural connections, induce dendritic sprouting, and lead to axonal outgrowth (Biernaskie \& Corbett, 2001; Bury \& Jones, 2002; Carmichael, Wei, Rovainen, \& Woolsey, 2001; Dancause et al., 2005; Qü et al., 1998; Schiene et al., 1996). Hence, it is plausible that similar neural adaptations occur in humans and manifest as altered brain activation as measured during functional imaging.

Although neuroplasticity is a credible and attractive explanation for altered movement-related brain activation poststroke, it is also possible that these observations are a result of changes in volitional motor commands and motor performance. Numerous studies provide evidence of altered kinetic, kinematic, and muscle activation profiles during paretic limb movement (Bowden, Balasubramanian, Neptune, \& Kautz, 2006; Cirstea \& Levin, 2000; Cruz, Waldinger, \& Kamper, 2005; Knutsson \& Richards, 1979). Such changes in motor commands and motor performance are difficult to control, and they confound the interpretation of functional brain imaging data (for review, see Baron et al., 2004; Krakauer, 2007). For example, cross-sectional studies that demonstrate altered brain activation during index finger opposition and hand gripping after stroke also report unintended movements of other digits and of the paretic wrist and elbow (Ward et al., 2003b; Weiller et al., 1992). Even the pioneering work of Miyai et al. (2002, 2003) that showed lateralized and elevated cortical activation during hemiparetic walking was unavoidably confounded by stroke-related changes in the spatiotemporal characteristics of the gait cycle, such as decreased swing phase symmetry. Indeed, neuroplastic adaptations to stroke, altered motor commands, and changes in motor performance are concurrent, interdependent processes that are difficult to distinguish. Hence, the meaning of altered movement-related brain activation after stroke is still unclear.

Recently, our group was compelled to consider explanations for altered brain activation during hemiparetic lower limb movement. We used functional magnetic resonance imaging (fMRI) to examine brain activation during pedaling in people with and without stroke (Promjunyakul, Schmit, \& Schindler-Ivens, 2015). People with stroke displayed reduced pedaling-related brain activation volume compared with age-matched controls. While changes in the structure or function of the strokeaffected brain could explain these findings, other possible contributors include alterations in motor commands and differences in pedaling performance. Specifically, asymmetries in pedaling performance, whereby the nonparetic limb performs more than half of the work of pedaling, are well documented after stroke and result in a more unilateral pedaling strategy compared with controls (Brown \& Kautz, 1998; Perell, Gregor, \& Scremin, 1998). This pedaling strategy may help explain our results, as brain activation is lower during unilateral compared with bilateral movement (Grefkes, Eickhoff, Nowak, Dafotakis, \& Fink, 2008; Noble, Eng, \& Boyd, 2014). Moreover, a post hoc, exploratory analysis revealed an association between brain activation volume and work accomplished by the paretic limb; volume increased with increased work from the paretic limb (Promjunyakul et al., 2015). 
The purpose of this study was to determine whether reduced pedaling-related brain activation poststroke can be explained by altered volitional motor commands and pedaling performance. We used fMRI to compare brain activation during volitional and passive pedaling. During volitional pedaling, subjects used their own voluntary muscle activity to pedal. During passive pedaling, they relaxed and allowed an experimenter to move their limbs. The passive condition eliminated motor commands to pedal and minimized between-group differences in pedaling performance (e.g., muscle activity, kinematics, symmetry). We hypothesized that, if volitional motor commands and pedaling performance contribute to reduced pedaling-related brain activation poststroke, then between-group differences would be reduced during passive compared with volitional pedaling. Additionally, brain activation would be different for volitional and passive pedaling. We also examined the feasibility of minimizing muscle activity for passive pedaling, which was necessary for testing our hypothesis. Portions of this work have been presented previously in abstract form (Cleland \& Schindler-Ivens, 2015).

\section{Materials and Methods}

\section{Subjects}

A total of 45 individuals (22 stroke and 23 control) were enrolled and screened for passive pedaling. All were free from (a) neurological disease or injury, except stroke; (b) contraindications to fMRI; and (c) medical conditions that could affect brain function or make it unsafe to pedal. Stroke and control subjects were matched for age - mean $(S D)$ : stroke: 62 (12) years, range = 33-83; control: 62 (12) years, range $=21-77 ; p=.93$. All stroke survivors had sustained their stroke at least 1.5 years prior to participating. There were 10 cortical, nine subcortical, and three unclassified strokes. Strokes were classified as cortical if the lesion included any part of the cerebral cortices. This classification included small, localized lesions of the gray matter and large lesions that extended into neighboring white matter. Strokes were classified as subcortical if they were restricted to regions outside the cerebral cortices. Stroke location was determined from MRI or, for subjects who did not advance to MRI, from the medical record. Subjects whose stroke location was unclassified were those who did not advance to MRI and had missing or incomplete medical records. Stroke subjects underwent the lower limb portion of the Fugl-Meyer assessment and the 8-m comfortable walk test. Subjects were considered hyperreflexic if their seated reflex score from the Fugl-Meyer assessment was $\leq 1$. See Table 1. All subjects provided written informed consent; procedures were approved by institutional review boards at Marquette University and the Medical College of Wisconsin.

\section{Pedaling Device}

The pedaling device has been described previously and validated for fMRI (Mehta, Verber, Wieser, Schmit, \& Schindler-Ivens, 2009, 2012; Promjunyakul et al., 2015). In brief, the pedaling device was a direct drive apparatus constructed of nonmetallic materials that provided a light frictional workload. An MRIcompatible rotary optical encoder (MR318; Micronor Inc., Newbury Park, CA) 


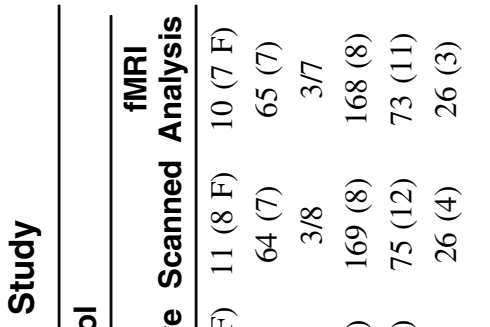

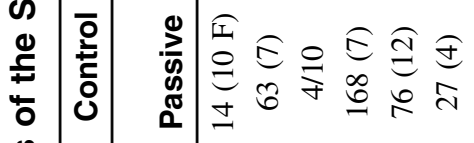

$$
\begin{aligned}
& \text { 曾 }
\end{aligned}
$$

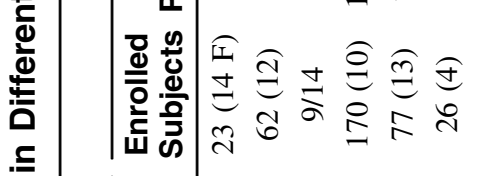

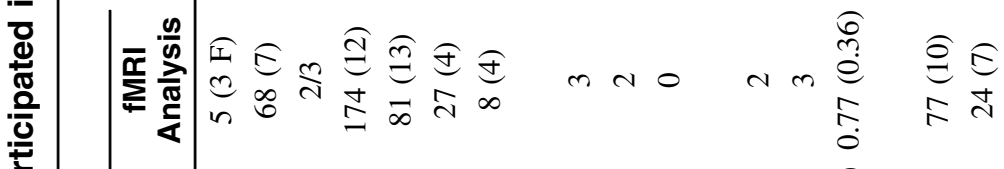

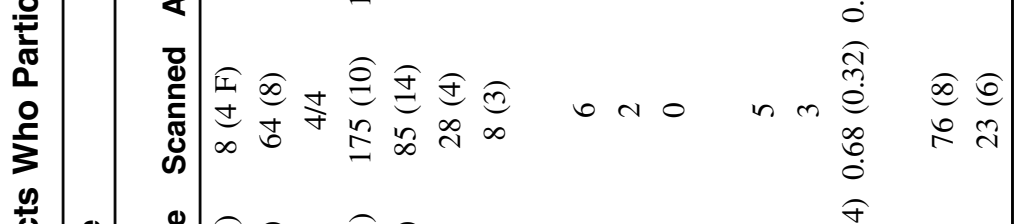

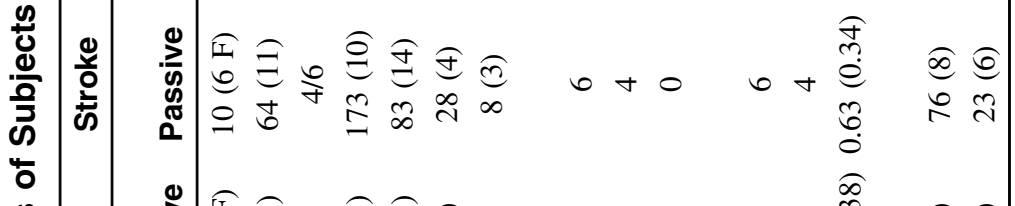

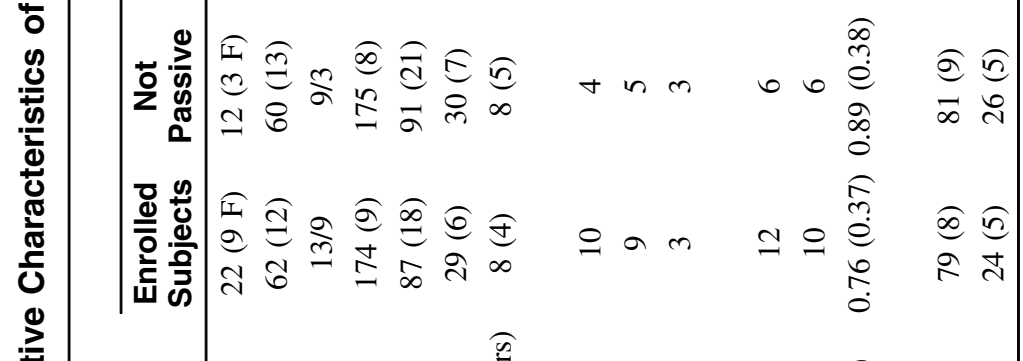

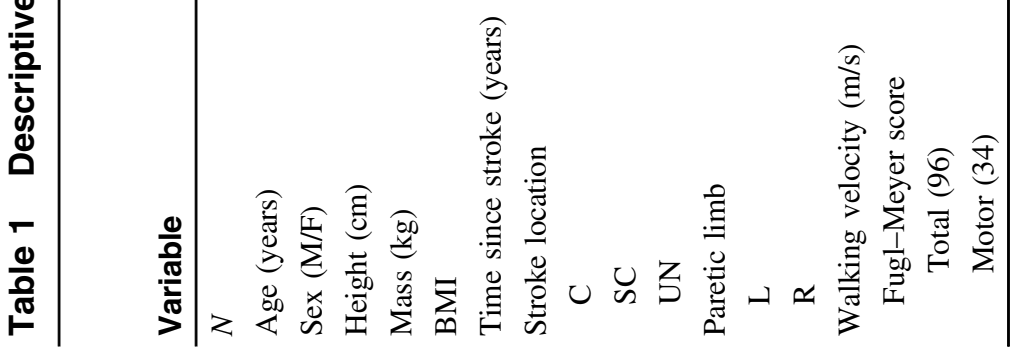




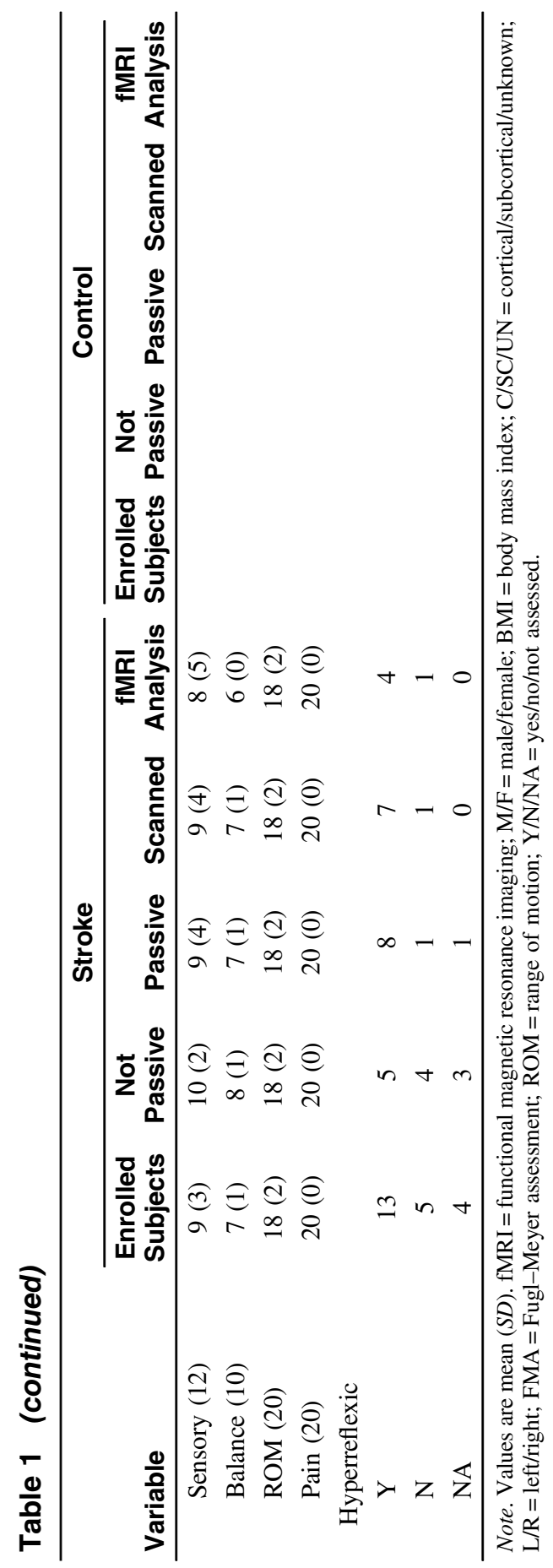


coupled to the crankshaft measured the position of the crankshaft across the pedaling cycle. Zero was defined for each crank arm as the position where the crank arm was parallel to the plinth and the foot was closest to the hip (top dead center, $0^{\circ}$ ). Signals from the encoder were carried to a controller unit (MR310; Micronor Inc.) via a fiber-optic cable and sampled to a desktop computer at $2000 \mathrm{~Hz}$ (Spike 2; Cambridge Electronic Design Ltd., Cambridge, UK).

\section{Feasibility of Passive Pedaling}

All subjects completed a familiarization session outside the fMRI environment to determine whether muscle activity could be minimized for passive pedaling (feasibility). Surface electrodes were placed bilaterally over tibialis anterior, medial gastrocnemius, rectus femoris, biceps femoris, and adductor magnus. Subjects lay supine on a plinth with their feet secured to the pedaling device. Volitional and passive pedaling were performed. During volitional pedaling, subjects were asked to use voluntary muscle activity to pedal. During passive pedaling, a member of the study team moved the pedals. Subjects were instructed to relax their legs and avoid assisting the study personnel. Each subject was given multiple opportunities to successfully complete the task over approximately 15 min of testing. In subjects with visible muscle activity, verbal cues were given during and after each trial about relaxing the active muscle(s). For example, if the medial gastrocnemius was active, subjects were given verbal feedback during and after the trial to focus on relaxing that muscle. As needed, another member of the study team provided manual support at the knees to prevent hip abduction and external rotation. Subjects wore solid ankle foot orthoses to stabilize the ankles and to minimize muscle activity in the dorsiflexors and plantar flexors. Ankle foot orthoses were also worn during the volitional condition.

For both passive and volitional pedaling, two trials of 30-s duration were recorded. An auditory pacing cue was used to maintain the desired rate of 45 revolutions per minute (RPM). The order in which passive and volitional conditions were introduced was counterbalanced. We also recorded electromyography (EMG) during quiet rest. Surface EMG was recorded with a bipolar, differential amplification system (Bagnoli-8; Delsys, Inc., Natick, MA), band-pass filtered $(20-450 \mathrm{~Hz})$, amplified $(1,000 \times)$, and passed to a 16-bit analog-to-digital convertor (Micro $1401 \mathrm{mk}$ II; Cambridge Electronic Design Ltd.). Signals were sampled to a desktop computer at $2000 \mathrm{~Hz}$ via data acquisition software (Spike 2; Cambridge Electronic Design Ltd.).

Adequate minimization of muscle activity during passive pedaling was determined per the following criteria. If muscle contractions were visually apparent, subjects were considered unable to achieve passive pedaling. For subjects with no visually apparent muscle contractions, EMG activity was examined offline using MATLAB (R2015b; The MathWorks, Inc., Natick, MA). Signals recorded during pedaling were rectified, smoothed (10-Hz fourth-order Butterworth low-pass filter), referenced to crank position, and averaged across cycles. Subjects were considered able to achieve passive pedaling if the mean EMG amplitude of each muscle was less than the mean $+4 S D$ of quiet rest. Using these criteria, subjects were classified as able (PASSIVE) or unable (NONPASSIVE) to minimize lower limb muscle activity for passive pedaling. PASSIVE subjects were invited for fMRI. 


\section{fMRI Procedures}

Of the 24 subjects who could perform passive pedaling, fMRI data from 15 subjects (five stroke and 10 control) were examined. Six subjects did not complete fMRI due to claustrophobia (one control), preexisting health conditions (one stroke, one control), loss to follow-up (one control), or bladder urgency (one stroke, one control). One stroke subject had no medically confirmed evidence of stroke. One stroke subject's head movement exceeded requirements ( $>4 \mathrm{~mm})$, and in one other stroke subject, no brain activation was detected with fMRI. See Table 1.

During fMRI, subjects lay supine on the scanner table with their feet secured to the pedals. The head was stabilized in the radio-frequency coil using a beaded vacuum pillow and foam padding. Chin and trunk straps were used to further reduce head and body movement. A strip of adhesive tape was placed on the forehead and secured to the outside of the coil to provide a sensory cue if the head moved. Ankle foot orthoses and manual support provided during familiarization were also applied during fMRI. Subjects performed passive and volitional pedaling in a block design consisting of three runs of each condition. Each run consisted of $18 \mathrm{~s}$ of rest followed by $20 \mathrm{~s}$ of pedaling and $20 \mathrm{~s}$ of rest, repeated five times. Auditory cues were used to maintain a pedaling rate of 45 RPM and to cue subjects to pedal or rest. Auditory cues were provided during pedaling and rest segments through MRI-compatible earbuds (model SRM 212; STAX Ltd., Kowloon, Hong Kong SAR). Condition order was counterbalanced.

MRI data were obtained using a 3.0-T magnetic resonance scanner (GE Healthcare, Milwaukee, WI) and a single-channel transmit/receive split head coil assembly (model 2376114; GE Healthcare). Functional images (T2*-weighted) were acquired using echoplanar imaging (repetition time [TR]: 2,000 ms, echo time: $25 \mathrm{~ms}$, flip angle: $77^{\circ}, 36$ contiguous slices in the sagittal plane, $64 \times 64$ matrix, $4 \mathrm{~mm}$ slice thickness, and field of view: $24 \mathrm{~cm}$ ). The resolution of the images was $3.75 \times 3.75 \times 4 \mathrm{~mm}$. Each run consisted of $109 \mathrm{TRs}$. Anatomical images (T1-weighted) were obtained half way through the scan session using a three-dimensional fast spoiled GRASS pulse sequence (TR: $8.2 \mathrm{~ms}$, echo time: $3.2 \mathrm{~ms}$, flip angle: $12^{\circ}, 256 \times 244$ matrix, resolution: $1 \mathrm{~mm}^{3}$, and field of view: $24 \mathrm{~cm}$ ). Audio cues were synchronized with magnetic resonance pulses using Presentation software (Neurobehavioral Systems, Inc., Berkeley, CA).

Analysis of Functional NeuroImages software was used to process fMRI data. Three-dimensional images were temporally aligned, and the first four TRs from each run were removed. All runs from a single condition were concatenated and registered to the passive run adjacent to the anatomical scan. General linear modeling was used to fit a canonical hemodynamic response function (boxcar function convolved with a gamma function) to the measured blood-oxygenationlevel dependent (BOLD) signal. Conventional fMRI signal processing for block designs in which the entire BOLD signal is fit to a canonical function may not be appropriate for detecting pedaling-related brain activation because limb and head motion may cause artifacts (Mehta et al., 2009). To address this potential confound, we fit only the rest portion of the BOLD time series after pedaling stopped to the canonical function (Figure 1). This approach is justified because the termination of the BOLD signal is delayed with respect to the termination of behavior (DeYoe, Bandettini, Neitz, Miller, \& Winans, 1994). Thus, data recorded during the rest 


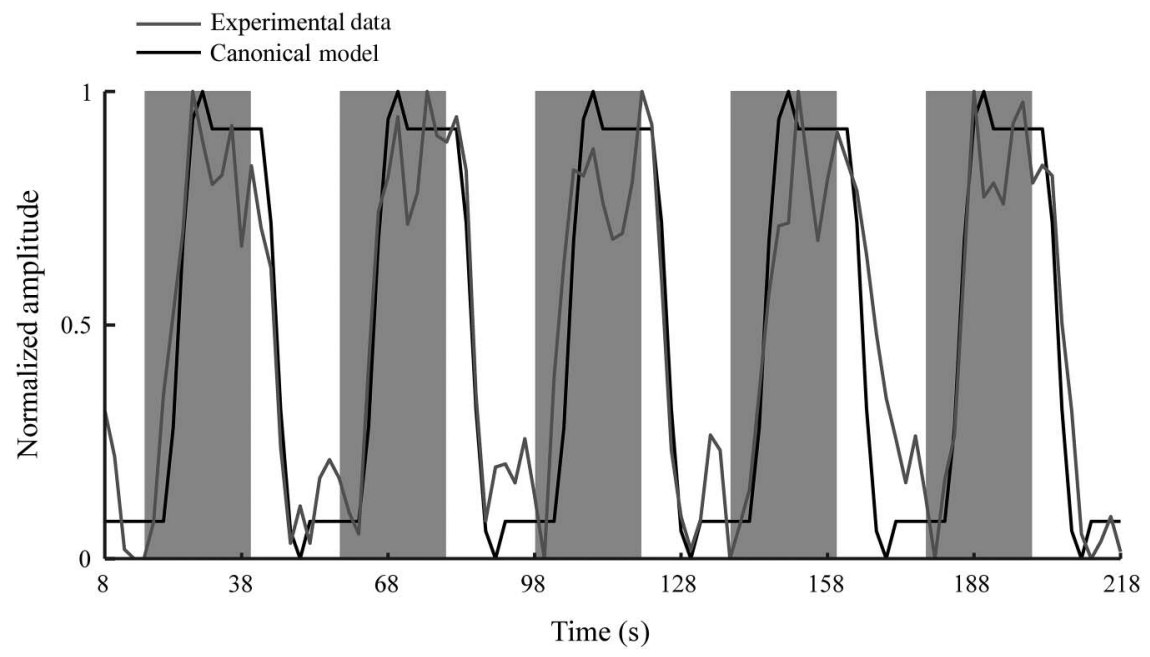

Figure 1 - Delayed nonmovement modeling technique. Experimental data from a single voxel in the sensorimotor cortex overlaid on the canonical hemodynamic response function. Gray shaded regions represent periods of time when the pedaling task was being performed, and white regions represent the rest periods. Data are shown for a single run with five pedaling blocks. The canonical hemodynamic response function (black line) was fit only to the portion of the BOLD response (gray line) obtained during rest periods. Note the delayed nature of the decline in the BOLD response associated with pedaling. BOLD = bloodoxygenation-level dependent.

period contain the end of the plateau portion of the BOLD signal, the declining phase of the BOLD signal, and baseline. This approach has been validated and used in prior work examining pedaling-related brain activation in individuals with and without stroke (Mehta et al., 2009, 2012; Promjunyakul et al., 2015). Head movement was used as a variable of no interest. Model fitting was performed in each subject's native coordinate system to avoid misregistration caused by conversion to standard space.

Noise smoothness was estimated using a spatial autocorrelation function, fit to a mixed model (Gaussian and monoexponential functions), and used to blur functional data. To identify significantly active voxels at a familywise error rate of $p<.05$, we used Monte Carlo simulation to set an appropriate cluster size for a given individual voxel at $p<.005$. Voxels outside of the brain, negatively correlated voxels, and voxels with percent signal change greater than 10 were ignored.

Anatomical landmarks defined in native space on a subject-by-subject basis were used to circumscribe the primary motor and sensory cortices (M1S1), Brodmann's area 6 (BA6), and cerebellum $(\mathrm{Cb})$ as previously described (Promjunyakul et al., 2015; Schmahmann et al., 1999; Wexler et al., 1997). These regions of interest (ROIs) were chosen because they were consistently activated across subjects. Quantitative measures of volume, intensity, and location of activation were extracted from each region and all regions combined. Volume 
was defined as the number of significantly active voxels in each region multiplied by voxel volume in microliters. These values were also normalized to the anatomic volume of the region. Intensity was defined as the average percent signal change from baseline. Location of activation was measured with laterality index (LI), defined as the difference in volume between the damaged and undamaged sides (stroke) or left and right sides (control) as a proportion of total volume on both sides of the brain. Positive LI values indicated activation more toward the damaged hemisphere or the left hemisphere for stroke and control subjects, respectively. In the $\mathrm{Cb}$, LI was inverted. Location of activation was also measured by the center of mass (COM) of activation in M1S1. Group data were obtained by extracting the volume, intensity, and LI values from ROIs defined in each subject's native space and averaging these values across all subjects.

Three types of head movement were estimated from volume registration performed in Analysis of Functional NeuroImages: (a) displacement-mean distance from registration point, (b) oscillation-mean variation around registration point, and (c) drift_ change in position from start to the end of each trial. For all three types, movement was determined in the $x$ (medial/lateral), $y$ (anterior/ posterior), and $z$ (inferior/superior) directions. Calculations were performed separately for each subject and for each condition.

\section{Statistical Tests}

Tests were performed in SPSS Statistics 22.0 (IBM Corporation, Armonk, NY). Effects were considered significant at $p<.05$.

Subject demographics. Descriptive characteristics between groups (control and stroke) were compared using Mann-Whitney $U$ (age, height, and body mass index [BMI]), chi-square (sex), and independent $t$ tests (mass). Chi-square and independent $t$ tests were also used to determine whether subjects who were scanned and whose fMRI data were used in analysis were different from the entire group in terms of sex, hyperreflexia, paretic limb, stroke location, age, height, mass, BMI, time since stroke, walking velocity, and Fugl-Meyer score (total, motor, sensory, balance, range of motion, and pain).

Feasibility of passive pedaling. In subjects who could perform passive pedaling, we examined the effect of condition on the mean EMG of each muscle; repeatedmeasures analysis of variance (ANOVA) with three levels of condition (passive, volitional, and rest) and five levels of muscle (tibialis anterior, medial gastrocnemius, rectus femoris, biceps femoris, and adductor magnus) was used. When an effect of condition was detected, post hoc pairwise comparisons were performed using Fisher's least significant difference. Data were collapsed across limbs, as we detected no significant between-limb differences $(p \geq .26)$. Numerous post hoc, exploratory analyses were used to identify factors associated with the ability to perform passive pedaling. Chi-square, Mann-Whitney $U$, and independent $t$ tests were used to examine the effects of sex, hyperreflexia, group, age, mass, BMI, Fugl-Meyer scores, time since stroke, and walking velocity. Logistic regression was used to examine the predictive value of sex, group, age, mass, and BMI on the ability to perform passive pedaling. Tests were performed in control and stroke groups and, where appropriate, on the two groups combined. 
Brain activation. Group and condition effects on brain activation volume, intensity, and LI of activation were examined using mixed-effect ANOVA with two levels of condition (passive and volitional), two levels of group (stroke and control), and three levels of region (M1S1, BA6, and Cb). Normalized and nonnormalized values for volume were examined. Effects of group and condition on COM were examined using mixed-effect ANOVA with two levels of condition (passive and volitional) and two levels of group (stroke and control).

Pedaling rate and head motion. Group and condition effects on pedaling rate and head motion were examined using mixed-effects ANOVA with two levels of condition (passive and volitional) and two levels of group (stroke and control). The ANOVA for head motion also used three levels of movement type (oscillation, drift, and displacement) and three levels of movement direction $(x, y$, and $z)$.

\section{Results}

\section{Subject Demographics}

Stroke and control groups were not different in age, height, BMI, or sex $(p \geq .07)$. Mass was higher in stroke than control subjects $(p=.03)$. For all characteristics examined, subjects involved in different steps of the study (familiarization, scanned, and fMRI analysis) were not different from all subjects enrolled $(p \geq .18)$. See Table 1.

\section{Feasibility of Passive Pedaling}

All enrolled subjects could perform volitional pedaling. However, only $53 \%$ of subjects (10 stroke [45\%] and 14 control [61\%]) could adequately minimize lower limb muscle activity for passive pedaling. As suggested by the representative data in Figure 2a, subjects who could perform passive pedaling displayed a marked reduction in EMG during passive compared with volitional pedaling. They also showed no visually apparent, phase-dependent modulation of EMG amplitude during the passive condition. Moreover, the EMG observed during passive pedaling resembled that recorded during quiet rest (Figure $2 b$ ). These observations were apparent in all five muscles examined and in both limbs of stroke and control subjects. Quantitative analyses of the EMG data support these conclusions. In subjects who could perform passive pedaling, mean $(S D)$ EMG across muscles and groups decreased from 0.0106 (0.0569) $\mathrm{mV}$ during volitional pedaling to $0.0024(0.0010) \mathrm{mV}$ during passive pedaling $(p<.001)$ and $0.0022(0.0011) \mathrm{mV}$ during quiet rest $(p<.001)$.

Most subjects who could not perform passive pedaling (20/21 [95\%]; 12 stroke and eight control) displayed a pattern of EMG activity in one or more muscles that resembled the EMG during volitional pedaling (see Figure 3). These subjects could reduce, but not eliminate, pedaling-related muscle activity. Some subjects (five stroke and five control) also displayed a relatively invariant level of EMG across the pedaling cycle that was greater than the mean $+4 S D$ of quiet rest. Because EMG amplitude in these muscles was not modulated across the pedaling cycle, we considered that these muscles were stabilizing their limbs during passive pedaling. Only one subject was considered NONPASSIVE because she showed this type of muscle activity alone. A few subjects (one stroke and three control) also 
(a) Representative examples
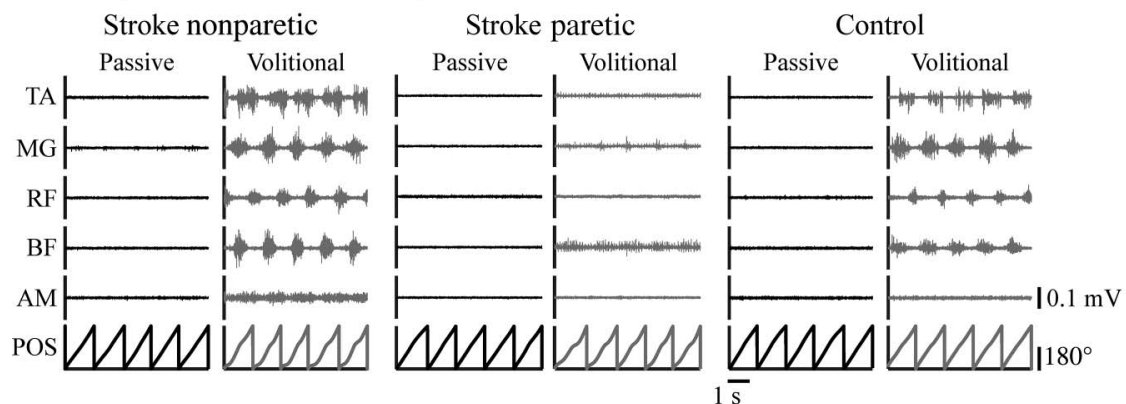

(b) Group data
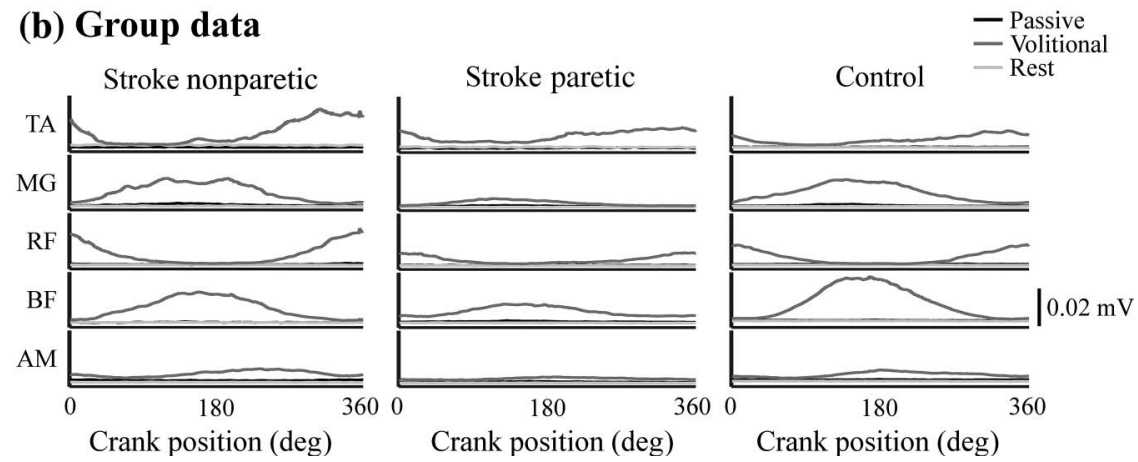

Figure 2 - Muscle activity during passive and volitional pedaling in PASSIVE subjects: representative examples and group averages. (a) Representative examples of EMG (raw and unprocessed) during passive and volitional pedaling in the nonparetic and paretic legs of one stroke subject and in the right leg of one control subject able to minimize muscle activity for passive pedaling. Data are shown for five revolutions. (b) Group average EMG (rectified, filtered, and ensemble-averaged) for the nonparetic, paretic, and control limbs from all subjects able to minimize muscle activity during passive pedaling. $\mathrm{EMG}=$ electromyography; $\mathrm{TA}=$ tibialis anterior; $\mathrm{MG}=$ medial gastrocnemius; $\mathrm{RF}=$ rectus femoris; $\mathrm{BF}=$ biceps femoris; $\mathrm{AM}=$ adductor magnus; $\mathrm{POS}=$ pedal position.

displayed EMG during periods of apparent muscle lengthening. Hence, it appeared that in these muscles reflex-generated muscle activity could not be fully eliminated for passive pedaling.

Sex was the only descriptive variable that was significantly associated with the ability to perform passive pedaling. The proportion of females in the PASSIVE group $(70 \%)$ was significantly higher than the proportion of males $\left(36 \% ; \chi^{2}=4.98\right.$, $p=.03)$, but there was no significant effect of group $\left(\chi^{2}=1.07, p=.30\right)$. Logistic regression analysis showed that sex was the only variable to make a significant contribution to the prediction of passive pedaling $\left(R^{2}=.15, p=.01\right)$. Moreover, when age, group, BMI, and mass were forced into the regression, the $F$ value on the full model decreased and the $p$ value increased. This observation suggests that the addition of descriptive variables other than sex had no positive effect and even impaired the relative predictive power of the model. Finally, as shown in Table 2, 
(a) Stroke

ST003 nonparetic

TA
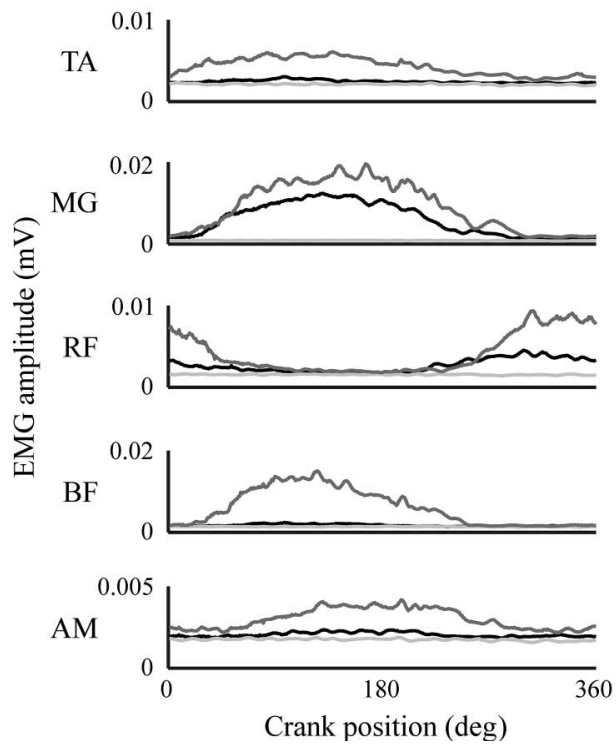

(b) Control
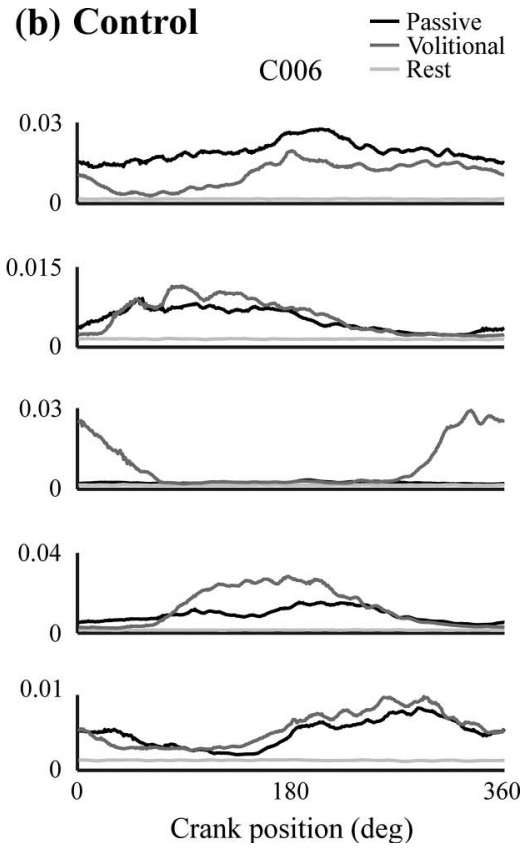

Figure 3 - Representative examples of NONPASSIVE stroke and control subjects. Examples from (a) one stroke and (b) one control subject who were classified as NONPASSIVE. Data shown are EMG (processed and ensemble averaged) during passive pedaling that were similar as during volitional pedaling. $\mathrm{EMG}=$ electromyography; $\mathrm{MG}=$ medial gastrocnemius; $\mathrm{RF}=$ rectus femoris; $\mathrm{BF}=$ biceps femoris; $\mathrm{AM}=$ adductor magnus.

there were no significant differences between PASSIVE and NONPASSIVE subjects with respect to age, mass, BMI, time since stroke, Fugl-Meyer scores, walking velocity, or hyperreflexia.

\section{Brain Activation, Passive Versus Volitional}

As illustrated by the examples in Figure 4, passive and volitional pedaling produced bilateral activation in M1S1, BA6, and $\mathrm{Cb}$ in both the stroke and control groups. In most subjects, activation in BA6 was limited to the supplementary motor area, and activation in the $\mathrm{Cb}$ was limited to the vermis and lobules IV, V, and VIII. Regardless of group or region examined, there was no significant difference in brain activation volume, intensity, or LI for passive compared with volitional pedaling. See Figure 5 and Table 3 . Normalized brain activation volume $(p=.34)$ and COM were also not different between conditions $(p=.14)$.

\section{Brain Activation, Stroke Versus Control}

Regardless of the region or condition examined, there were no between-group differences (stroke vs. control) in activation volume or LI. Normalized brain 
Table 2 Differences Between PASSIVE and NONPASSIVE Subjects

\begin{tabular}{lcccc}
\hline & PASSIVE & NONPASSIVE & $\boldsymbol{t}$ Value & $\boldsymbol{p}$ Value \\
\hline All & & & & \\
Age & $64(9)$ & $61(14)$ & 0.82 & .42 \\
Mass & $79(13)$ & $85(20)$ & -1.21 & .30 \\
BMI & $27(4)$ & $28(6)$ & -0.28 & .78 \\
Control & & & & \\
Age & $63(7)$ & $61(17)$ & 0.44 & .67 \\
Mass & $76(12)$ & $77(16)$ & -0.09 & .93 \\
BMI & $27(4)$ & $25(4)$ & 1.08 & .29 \\
Stroke & & & & \\
Age & $64(11)$ & $60(13)$ & 0.68 & .50 \\
Mass & $83(14)$ & $91(21)$ & -1.11 & .28 \\
BMI & $28(4)$ & $30(7)$ & -0.82 & .42 \\
Time since stroke & $8(3)$ & $8(5)$ & 0.29 & .78 \\
Walking velocity & $0.63(0.34)$ & $0.89(0.38)$ & -1.53 & .15 \\
Fugl-Meyer score & & & & \\
Total & $76(8)$ & $81(9)$ & -1.28 & .22 \\
Motor & $23(6)$ & $26(5)$ & -0.97 & .35 \\
Sensory & $9(4)$ & $10(2)$ & -0.85 & .41 \\
Balance & $7(1)$ & $8(1)$ & -1.98 & .14 \\
ROM & $18(2)$ & $18(2)$ & -0.31 & .76 \\
Pain & $20(0)$ & $20(0)$ & 0 & $>.99$ \\
\hline & & & $\chi^{2}$ & $\boldsymbol{p}$ Value \\
\hline Hyperreflexia & $5(89 \%)$ & $5(56 \%)$ & 2.49 & .11 \\
\hline
\end{tabular}

Note. Data are shown for all subjects, the control group alone, and the stroke group alone. Values for age (years), mass $(\mathrm{kg})$, and BMI $\left(\mathrm{kg} / \mathrm{m}^{2}\right)$ are shown for both groups. Values for time since stroke (years), walking velocity (m/s), Fugl-Meyer scores, and hyperreflexia are also shown for the stroke group. Values for hyperreflexia are count $(\%)$. All other values are mean $(S D)$. BMI = body mass index; $\mathrm{ROM}=$ range of motion

activation volume was also not different between groups $(p=.55)$. In contrast, the intensity of pedaling-related brain activation (collapsed across conditions) was higher in the stroke group compared with the control group for all active regions $(p<.001)$. There were also no Group $\times$ Condition interactions (intensity: $p=.13$; volume: $p=.95$; LI: $p=.36$ ). See Table 3 .

\section{Pedaling Rate and Head Motion}

During fMRI, pedaling was performed at the desired rate, as shown by a mean $(S D)$ rate of 45 (3) RPM that did not differ across conditions or groups (Condition: $p=.19$; Group: $p=.42$; Condition $\times$ Group: $p=.38$ ). During familiarization, pedaling rate was not different between groups, 44.9 (1.8) RPM, $p=.08$. However, 
(a) Stroke
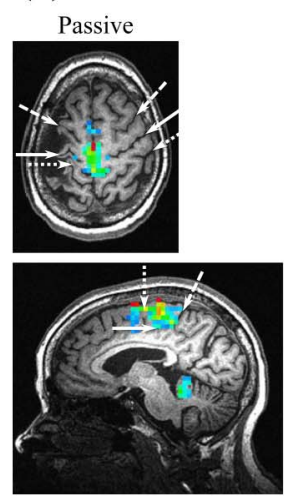

Volitional
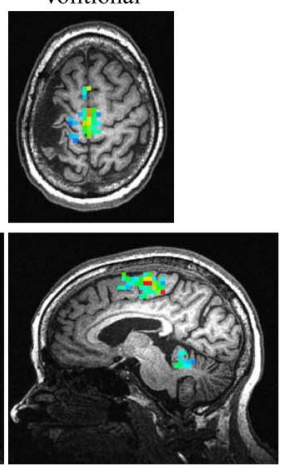

(b) Control
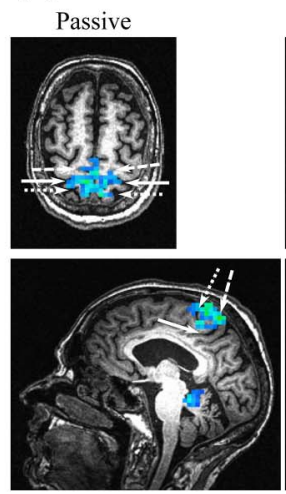

Volitional
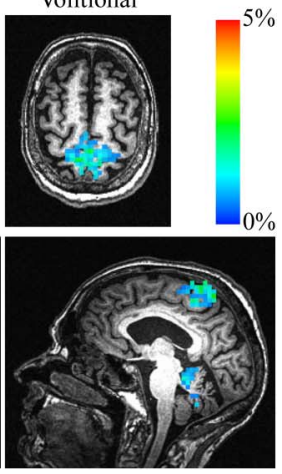

Figure 4 - Representative examples of pedaling-related brain activation. fMRI activation during pedaling in a (a) stroke and (b) control subject. A single axial and sagittal slice is shown for each subject to demonstrate the brain activation during passive and volitional pedaling. The same slices are depicted for passive and volitional pedaling. Color scale represents percent signal change compared with rest $(0-5 \%)$. White arrows indicate anatomical landmarks. In the axial plane: long dash $=$ precentral sulcus; solid line $=$ central sulcus; short dash = postcentral sulcus. In the sagittal plane: long dash = marginal sulcus; solid line $=$ cingulate sulcus; short dash $=$ paracentral sulcus. $\mathrm{fMRI}=$ functional magnetic resonance imaging.

the rate of passive pedaling, 44.2 (1.0), was lower than the rate of volitional pedaling, 45.6 (2.1), with an effect size of 1.4 RPM and $p<.001$.

Head motion was successfully minimized during fMRI. Subjects included in fMRI analysis displayed $0.22(0.20) \mathrm{mm}$ of oscillation, $0.58(0.64) \mathrm{mm}$ of displacement, and $0.46(0.52) \mathrm{mm}$ of drift. Values did not differ across conditions or groups (Condition: $p=.19$; Group: $p=.62$; Condition $\times$ Group: $p=.22$ ).

\section{Discussion}

This study provides three novel discoveries with important implications for understanding neural control of leg movement after stroke. First, we found that passive pedaling did not reduce between-group differences (stroke vs. control) in pedaling-related brain activation. Second, brain activation in people with and without stroke was not significantly different between passive and volitional pedaling. Together, these observations suggest that altered volitional motor commands and pedaling performance are unlikely to account for reduced pedalingrelated brain activation poststroke, as reported by Promjunyakul et al. (2015). Instead, this phenomenon may be due to loss of structural connectivity among brain regions, exaggerated cortical inhibition, increased reliance on spinal and brainstem pathways for rhythmic leg movement, or poor sensorimotor integration. These mechanisms require further study. The lack of difference between brain activation during passive and volitional pedaling also suggests that sensory signals 
(a)

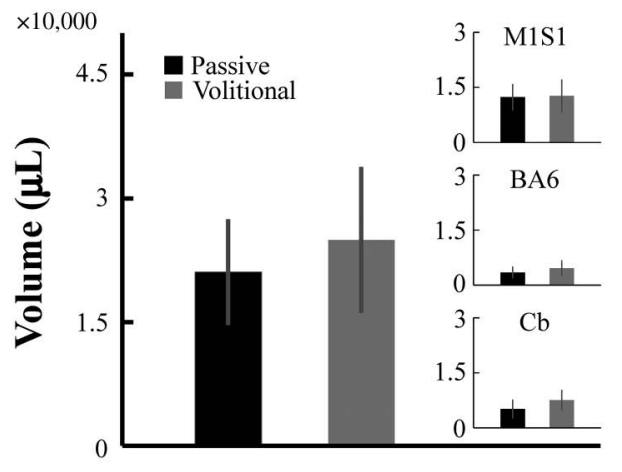

(b)

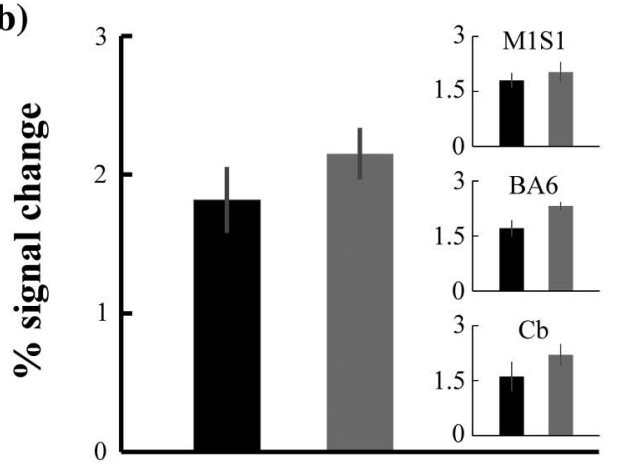

Control

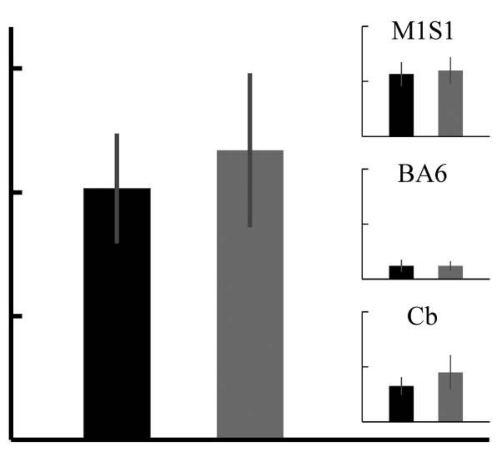

M1S1

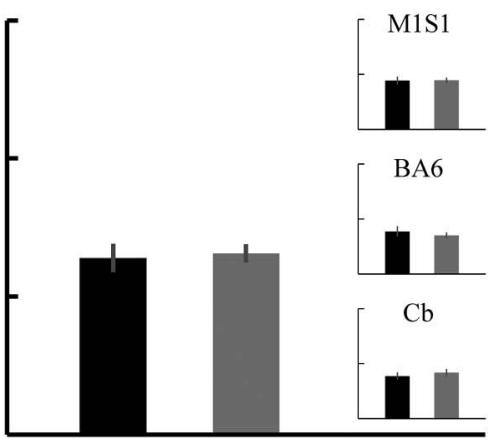

(c)

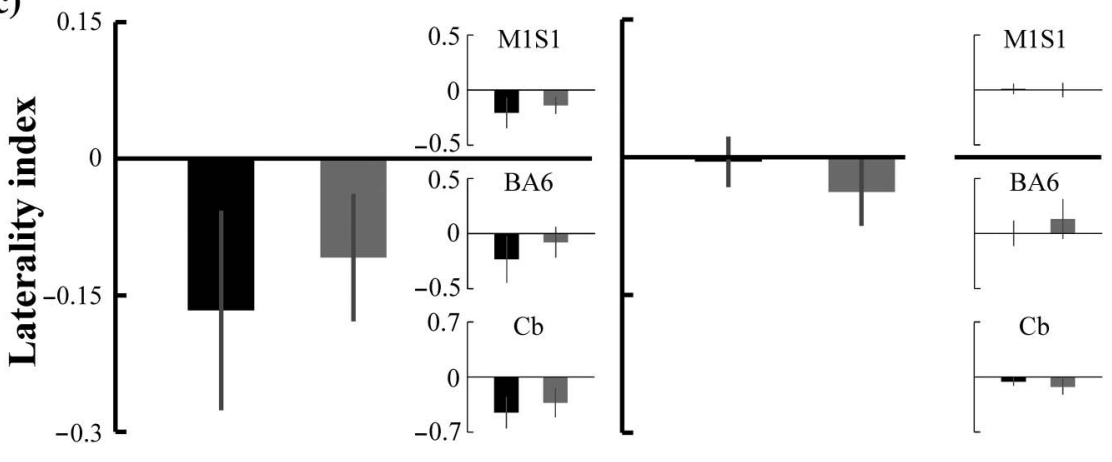

Figure 5 - Group average pedaling-related brain activation for all active brain regions. (a) Volume, (b) intensity, and (c) laterality index of brain activation. Large bar graphs show activation values for all active brain regions. Inset bar graphs show activation values for the indicated region of interest. Each dependent variable was extracted from native space on a subject-by-subject basis and then averaged for each group and for each condition. Volume, intensity, and laterality index were similar for both groups when comparing passive and volitional pedaling. Error bars are SE. M1S1 = primary motor and sensory cortices; BA6 $=$ Brodmann's area $6 ; \mathrm{Cb}=$ cerebellum. 


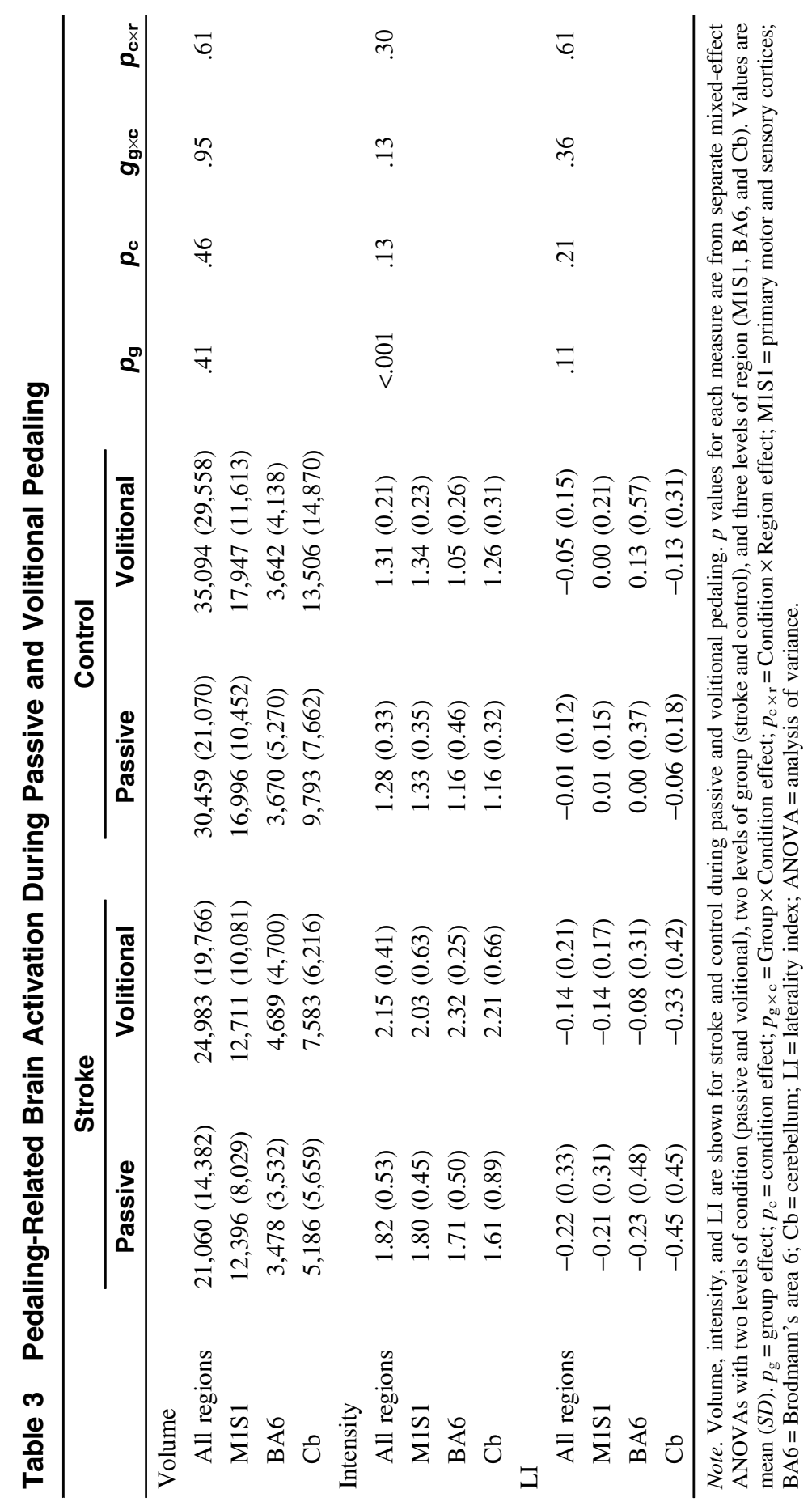


from the moving limbs make an important contribution to pedaling-related brain activation in people with and without stroke. Third, our data demonstrate that it is not uncommon for stroke and control subjects to have difficulty minimizing lower limb muscle activity for passive pedaling. Passive pedaling may require processes that are more developed in some individuals than others, such as alteration of descending drive, inhibition of reflexes, and inhibition of pattern generating circuits.

\section{Motivation and Limitations}

This study was motivated by our prior work demonstrating that the volume of pedaling-related brain activation is reduced in people poststroke compared with age-matched controls (Promjunyakul et al., 2015). Unlike our prior work, the present study found no significant difference in pedaling-related brain activation volume between the stroke and control groups. The absence of a significant group effect on volume is a limitation and raises the possibility that there is no real reduction in pedaling-related brain activation volume after stroke. However, several pieces of evidence suggest otherwise. In the present study, the volume of activation across all regions was $29 \%$ lower in the stroke group than in the control group. This reduction is consistent with the $27 \%$ reduction reported previously that reached statistical significance (Promjunyakul et al., 2015). Hence, it is likely that the lack of significance reported here was due to a small sample and low statistical power, not a true absence of a between-group difference. The intensity data provide further evidence that sampling may account for inconsistencies between studies. The present study found significantly higher pedalingrelated brain activation intensity in the stroke group compared with the control group. In our prior publication (Promjunyakul et al., 2015), there was a trend toward higher intensity activation poststroke that was not statistically significant. These observations suggest that interindividual variation may have contributed to disparate results across studies. Larger samples would be useful in future work to increase statistical power and to better represent the full range of responses within the population. In this study, our sample was constrained by strict criteria for passive pedaling that were necessary for eliminating motor commands to pedal and minimizing between-group differences in pedaling performance. With these limitations in mind, we discuss the implications of our results in the sections that follow.

\section{Influence of Volitional Motor Commands and Pedaling Performance on Brain Activation Poststroke}

Plausible explanations for reduced pedaling-related brain activation poststroke are not limited to neuroplasticity. Altered volitional motor commands and pedaling performance may contribute. Unlike control subjects who use both limbs equally to pedal (Ambrosini, Ferrante, Ferrigno, Molteni, \& Pedrocchi, 2012; Brown \& Kautz, 1998), people with stroke typically exhibit an asymmetric pedaling strategy in which the nonparetic limb contributes more than half the work of pedaling (Brown \& Kautz, 1998; Perell et al., 1998). This strategy is characterized by abnormal muscle activation patterns, kinetics, and kinematics that may be driven 
by volitional motor commands that are different from normal. These phenomena occur at the same time and may be interdependent with neuroplastic adaptations. Thus, it is difficult to separate the contributions of neuroplasticity, altered motor commands, and pedaling performance to changes in pedaling-related brain activation.

We are not the first to consider the effects of task performance on movementrelated brain activation poststroke. Levin, Kleim, and Wolf (2009) included this issue in a contemporary review highlighting the importance of altered movement patterns after stroke. Other commentaries and position papers have also included altered task performance as a limiting factor in the interpretation of fMRI data (Baron et al., 2004; Krakauer, 2007). To examine this issue, subjects performed passive and volitional pedaling while we examined brain activation with fMRI. Passive pedaling eliminated motor commands to pedal and minimized betweengroup differences in pedaling performance (e.g., muscle activity, kinematics, symmetry), providing insight into how motor commands and pedaling performance may influence brain activation. We reasoned that if motor commands and pedaling performance have an important influence on brain activation poststroke, then between-group differences in the volume, intensity, and/or location of brain activation would be reduced during passive compared with volitional pedaling. Moreover, brain activation would be different during passive compared with volitional pedaling.

Contrary to our prediction, our data suggest that motor commands and pedaling performance do not produce significant changes in brain activation poststroke. Despite eliminating motor commands to pedal and minimizing between-group differences in muscle activity, kinematics, and symmetry, passive pedaling did not minimize differences in brain activation volume, intensity, or LI between the stroke and control groups. On average, the volume of brain activation in the stroke group was $71 \%$ of control during volitional pedaling and $69 \%$ of control during passive pedaling. Intensity values for the stroke group were $164 \%$ of control during volitional and $142 \%$ of control during passive pedaling. For LI, values for the stroke and control groups were -0.14 and -0.05 during volitional pedaling and -0.22 and -0.01 during passive pedaling, respectively. Furthermore, we saw no significant difference in the volume, intensity, or LI of brain activation during passive compared with volitional pedaling. We also found no evidence of a shift in the COM of activation when comparing passive to volitional pedaling. Taken together, these observations suggest that whether pedaling is performed volitionally or passively, brain activation remains largely unchanged. If such a robust manipulation of motor commands and pedaling performance does not alter brain activation, then less substantial differences in motor commands and pedaling performance, such as asymmetric work output, are unlikely to explain stroke-related decreases in brain activation volume that have been described (Promjunyakul et al., 2015).

The absence of significant differences in brain activation between volitional and passive pedaling also suggests that sensory signals from the moving limbs make an important contribution to pedaling-related brain activation in people with and without stroke. This observation is consistent with a prior study from our lab that used fMRI to demonstrate that cortical activation in young adults without stroke was not different during passive and volitional pedaling (Mehta et al., 2012). 
Moreover, Christensen et al. (2000) recorded brain activation volume and peak regional cerebral blood flow (rCBF) with positron emission tomography (PET) during passive and volitional pedaling in adults without stroke. Despite differing from our study in imaging modality (PET vs. fMRI) and pedaling rate (60 vs. 45 RPM), Christensen's results are consistent with ours. They reported no betweencondition differences in activation volume or peak $\mathrm{rCBF}$ in the primary sensory area, supplementary motor area, or cerebellar vermis. The only significant difference between conditions was a $12-14 \%$ increase in $\mathrm{rCBF}$ in M1 during volitional compared with passive pedaling. While not reaching statistical significance, the present paper and our prior work (Mehta et al., 2012) reveal a tendency for brain activation volume in M1 to be larger (13-14\%) during volitional compared with passive pedaling. Perhaps the lack of significance in our work is related to sample size or our use of mean intensity, not peak rCBF. Overall, the observations reported here provide further evidence that much of the brain activation during pedaling may not be related to the volitional commands or muscle activity, but rather to monitoring sensory information to respond to perturbations or to maintain, reinforce, or shape ongoing motor output.

A largely sensory contribution to pedaling-related brain activation, particularly for motor areas of the brain, may seem counterintuitive. However, many studies have found that brain activation in neurologically intact individuals is not different or is minimally different during passive and volitional movements of the finger, hand, wrist, elbow, shoulder, toe, ankle, knee, or hip (Blatow et al., 2011; Boscolo Galazzo et al., 2014; Guzzetta et al., 2007; Kocak, Ulmer, Sahin Ugurel, Gaggl, \& Prost, 2009; Onishi et al., 2013; Terumitsu, Ikeda, Kwee, \& Nakada, 2009; Weiller et al., 1996). In people with stroke, passive and volitional movements of the finger and ankle are associated with the same brain activation (Alary et al., 1998; Enzinger et al., 2008). Other studies in humans support an influence of sensory input on brain activation. When anesthesia is used to block muscle afferent feedback during static and dynamic contractions, a large decrease in brain activation is observed in humans (Friedman, Friberg, Mitchell, \& Secher, 1991; Friedman, Friberg, Payne, Mitchell, \& Secher, 1992).

Alternatively, passive pedaling may engage different motor commands (e.g., commands to relax the legs) that produce a similar fMRI response as the volitional command to pedal. Similarly, because fMRI cannot distinguish between excitation and inhibition (Logothetis, 2008), volitional and passive pedaling may differentially activate excitatory and inhibitory circuits without changing total cortical activation measured with fMRI. For example, when individuals relax from a contraction, there is an increase in the magnitude of short-interval intracortical inhibition (Motawar, Hur, Stinear, \& Seo, 2012) but no change in cortical activation (Toma et al., 1999). During response inhibition, cortical excitability decreases and and short-interval intracortical inhibition increases (Coxon, Stinear, \& Byblow, 2006). Passive pedaling may involve analogous alterations in the balance of cortical excitation and inhibition that decrease descending drive to spinal motor neurons without changing brain activation as measured with fMRI.

Limitations in the measurement properties of fMRI may have contributed to our results. Passive pedaling may induce enough neural activation to saturate the blood flow response such that additional neural activation caused by motor commands fails to increase the fMRI signal. Evidence for this hypothesis comes 
from Reddy, Floyer, Donaghy, and Matthews (2001), who found no difference in brain activation, as measured with fMRI, during volitional and passive finger movements in neurologically intact individuals. In people with sensory neuropathy, volitional movement produced activation that was indistinguishable from the neurologically intact group, whereas passive movement produced no measurable brain activation (Reddy et al., 2001). These observations suggest that both sensory and motor signals contribute to brain activation, but they do not have an additive effect on the fMRI signal.

Finally, our results could be influenced by a sample that does not represent the population. Forty-seven percent of our sample could not achieve passive pedaling according to our strict criteria; these subjects did not undergo fMRI. Individuals who could achieve passive pedaling might be different from those who could not. Specifically, they might be able to inhibit muscle activity through an active process. As discussed above, active inhibition may produce similar fMRI signals as volitional commands to pedal. Detracting from this explanation is prior work showing no difference in brain activation during passive and volitional movements (including pedaling) with minimal or nonexistent criteria for achieving the passive condition (see Minimizing Muscle Activity for Passive Pedaling subsection). These observations suggest that our results may generalize to the broader population, including individuals who cannot completely relax for passive pedaling.

\section{Explanations for Reduced Pedaling-Related Brain Activation Poststroke}

Finding no compelling evidence for altered motor commands and pedaling performance as an explanation for reduced brain activation poststroke, we continue to consider alternative explanations. With respect to anatomical contributions, reduced pedaling-related brain activation volume could be caused by the loss of viable brain tissue, as all stroke survivors suffered tissue loss. However, no stroke survivors had lesions affecting the leg area of M1S1, and only one subject had a lesion affecting the $\mathrm{Cb}$. Thus, as in our prior work, brain activation was lacking in apparently vital regions that are typically involved in pedaling (Promjunyakul et al., 2015). Therefore, stroke may cause a fundamental change in the structure or function of the brain. In the following paragraphs, we consider several possibilities that could be tested in future work.

Reduced activation volume in anatomically intact brain regions could be explained by impaired white matter connectivity (Kalinosky, Schindler-Ivens, \& Schmit, 2013; Zhang et al., 2016). All stroke subjects examined had some white matter damage, which could reduce the effects of signals from intact portions of the brain. Moreover, unless these signals find other pathways, intact brain regions may stop firing for lack of effects on their targets. Similarly, loss of structural connectivity could reduce the amount of sensory input reaching the cortex, which may be a major source of pedaling-related cortical activation, as discussed previously (Christensen et al., 2000; Mehta et al., 2012). Although plausible, these hypotheses require further study. Contrary to these assertions, others have suggested that reduced connectivity among motor areas of the brain leads to overactivity of cortical tissue (Hamzei, Dettmers, Rijntjes, \& Weiller, 2008). 
Elevated cortical inhibition could also contribute to reduced pedaling-related brain activation volume poststroke. Previous work has demonstrated reduced excitability of the lesioned hemisphere (Byrnes, Thickbroom, Phillips, Wilson, \& Mastaglia, 1999; Liepert, Uhde, Gräf, Leidner, \& Weiller, 2001). Exaggerated inhibition could be intrinsic to the lesioned hemisphere, or it could be due to interhemispheric inhibition whereby transcallosal output from the undamaged hemisphere inhibits M1 of the damaged hemisphere (Murase, Duque, Mazzocchio, \& Cohen, 2004; Traversa, Cicinelli, Pasqualetti, Filippi, \& Rossini, 1998). These phenomena manifest as lateralized activation toward the intact side of the brain. Our data are in line with this explanation, as there was a tendency for lateralization of brain activation toward the undamaged cortex and $\mathrm{Cb}$, although this observation did not reach statistical significance.

It is also possible that reduced pedaling-related brain activation volume after stroke is due to enhanced reliance on the spinal cord and/or brainstem for lower limb movement. Kautz, Patten, and Neptune (2006) found that pedaling with one leg induces rhythmic muscle activity in the contralateral leg in both stroke and control subjects. There was a greater induction of activation in the contralateral, nonmoving limb in the stroke group compared with the control group, particularly in those with greater impairments (Kautz et al., 2006). This result suggests that stroke survivors may have a greater reliance on spinal cord pathways for pedaling than controls. Perhaps the reduced cortical activation observed in stroke survivors is adequate for initiating pedaling, after which the maintenance of ongoing movement occurs in the brainstem and/or spinal cord. The result may be the unsophisticated and inflexible pattern of leg movement that is characteristic of hemiparesis.

Finally, impaired somatosensory integration in the cortex may also contribute to reduced pedaling-related brain activation after stroke. During passive wrist movement, stroke survivors with severe sensory impairment have reduced somatosensory evoked responses as measured by electroencephalography (Vlaar et al., 2017). Similarly, several studies have demonstrated that somatosensory evoked potentials are absent in stroke survivors who experience poor motor recovery (Feys, Van Hees, Bruyninckx, Mercelis, \& De Weerdt, 2000; La Joie, Reddy, \& Melvin, 1982). These studies suggest that there are impairments in the transmission of sensory information to the cortex or impairments in the cortical integration of sensory information after stroke. These deficits might result in decreased pedalingrelated brain activation after stroke.

\section{Minimizing Muscle Activity for Passive Pedaling}

The other major discovery from this study was that a large proportion of subjects (55\% stroke and 39\% control) had difficulty minimizing lower limb muscle activity for passive pedaling. The proportion of subjects unable to perform passive pedaling was surprisingly large given that few prior studies excluded subjects for failing to perform a passive condition. Our rate of exclusion may be due to the complexity of the task; subjects were required to relax multiple muscles across multiple joints of both limbs. Most other studies of passive movement were limited to a single joint, did not record muscle activity, and/or did not provide quantitative measures of EMG (Alary et al., 1998; Blatow et al., 2011; Boscolo 
Galazzo et al., 2014; Christensen et al., 2000; Enzinger et al., 2008; Guzzetta et al., 2007; Jain, Gourab, Schindler-Ivens, \& Schmit, 2013; Kocak et al., 2009; Mehta et al., 2012; Onishi et al., 2013; Terumitsu et al., 2009; Weiller et al., 1996). Our criteria for minimizing muscle activity were rigorous, requiring the near absence of EMG. The presence of pedaling-related EMG despite instruction and effort to remain passive raises questions about the physiological mechanisms underlying ability to perform passive movement. There may be interindividual differences in the ability to alter descending drive, inhibit spinal reflexes, or inhibit pattern generating activity.

To minimize muscle activity for passive pedaling, individuals may need to reduce excitatory descending drive to spinal motor neurons. The ability to do so may be mediated by intracortical inhibition, which may differ among individuals. Jain et al. (2013) and Yamaguchi, Fujiwara, Liu, and Liu (2012) suggest that passive pedaling may require a greater level of intracortical inhibition than volitional pedaling. Using electroencephalography, Jain et al. (2013) found that passive pedaling induced less beta desynchronization than volitional pedaling. As prior work has established an inverse relationship between beta desynchronization and intracortical inhibition (Takemi, Masakado, Liu, \& Ushiba, 2013), this observation suggests that passive pedaling requires a higher level of intracortical inhibition than volitional pedaling. Yamaguchi et al. (2012) used transcranial magnetic stimulation to evaluate intracortical inhibition before and after passive and volitional pedaling. They found that intracortical inhibition was the same before and after passive pedaling, but lower after volitional pedaling. Again, this observation suggests that passive pedaling may involve intracortical inhibition. Work from Coxon et al. (2006) and Motawar et al. (2012) provides further support for these conclusions. These authors have suggested that intracortical inhibition is important for relaxing from a volitional contraction or preventing a prepared movement.

Stretch reflexes may be responsible for residual EMG during passive pedaling. Typically, monosynaptic reflexes are dramatically reduced by both passive and volitional pedaling (Brooke, McIlroy, \& Collins, 1992; Brooke, Misiaszek, \& Cheng, 1993; Fuchs, Sanghvi, Wieser, \& Schindler-Ivens, 2011; Larsen, Voight, \& Grey, 2006; McIlroy, Collins, \& Brooke, 1992; Motl, Knowles, \& Dishman, 2003; Schindler-Ivens et al., 2008). The mechanism responsible for reflex suppression, presynaptic inhibition of primary afferent terminals (Brooke et al., 1992; McIlroy et al., 1992), may be better developed in some individuals than others. Indeed, Group Ia reflex suppression during pedaling is severely reduced in some people with stroke, but near normal in other people with stroke (Fuchs et al., 2011; Schindler-Ivens et al., 2008). Even in able-bodied individuals, inhibition of Group Ia afferent input is affected by training (Nielsen, Crone, \& Hultborn, 1993). Yet, it is unlikely that hyperexcitable reflexes significantly contribute to our results because few control $(n=3)$ and stroke $(n=1)$ subjects displayed EMG during lengthening phases of passive pedaling. Additionally, 20/21 (95\%) of NONPASSIVE subjects had at least one muscle that showed activation like that seen during volitional pedaling.

Some subjects may have difficulty suppressing sensory input that influences the pattern-generating circuits that contribute to rhythmic movement. It is well established that sensory input activates spinal pattern-generating circuits in nonhuman animals (Prochazka \& Ellaway, 2012) and influences the timing and 
amplitude of human rhythmic motor output (Grey, Nielsen, Mazzaro, \& Sinkjaer, 2007; Sinkjaer, Andersen, Ladouceur, Christensen, \& Nielsen, 2000; Stephens \& Yang, 1999; Verschueren, Swinnen, Desloovere, \& Duysens, 2002; Yang, Stein, \& James, 1991). To achieve passive pedaling, it may be necessary to suppress sensory input from muscle length, velocity, or load receptors or to suppress the output of pattern-generating circuits influenced by these sensory signals. The ability to do so may vary among individuals as described previously for monosynaptic reflex suppression. This mechanism could account for cases in which the muscle activation was reduced during passive compared with volitional pedaling, but the pattern of EMG was the same across conditions. Ninety-five percent of subjects who were unable to achieve passive pedaling displayed this EMG pattern in at least one muscle.

Finally, we cannot rule out interindividual differences in EMG signal detection as a possible explanation for differences in the ability to achieve passive pedaling. Fewer males than females could achieve passive pedaling. This finding may reflect better EMG signal detection in males, who typically have less body fat and more lean mass than women, including in the lower body (Power \& Schulkin, 2008). However, post hoc analyses of descriptive data suggest that differences in body composition may not explain the observed sex differences. We found no differences between males and females with respect to mass or BMI. Moreover, PASSIVE individuals had lower values for mass and BMI compared with NONPASSIVE individuals. Additionally, our logistic regression analysis indicated that sex, but not mass or BMI, was related to ability to perform passive pedaling. Therefore, we cannot rule out the possibility that sex influences neuromuscular control of passive movement or inhibition of rhythmic movement. However, we are unaware of evidence of sex differences in the ability to alter descending drive, alter the excitability of spinal reflexes, or inhibit patterngenerating circuits.

\section{Additional Limitations}

Despite our strict criteria during passive pedaling, a limitation of this study is that EMG activity was not measured during the fMRI scanning session. Differences in environment between the familiarization and scanning sessions could lead to changes in the ability to perform passive pedaling. Consequently, the lack of difference between brain activation during passive and volitional pedaling may be a result of the presence of muscle activity during passive pedaling. However, we did not experience any evidence of muscle contraction during passive pedaling in the MRI scanner. Our fMRI results are limited by a small sample size, particularly in the stroke group where five subjects were included. The small sample increases the risk of Type II error and limits generalizability to the population. However, the strict criteria that resulted in the small sample also allowed us to maximize differences between volitional and passive pedaling and minimize between-group differences in motor commands and pedaling performance. The lack of visual or auditory biofeedback about muscle activity during passive pedaling may have contributed to the large number of individuals who were unsuccessful at the task. Future studies may benefit from more extensive training with biofeedback to help participants achieve passive pedaling. 


\section{Conclusions}

This study found that between-group differences in brain activation were not reduced during passive compared with volitional pedaling and that brain activation was not different between these conditions. These results suggest that factors besides altered volitional motor commands and pedaling performance may contribute to a reduction in pedaling-related brain activation poststroke, including loss of structural connectivity, exaggerated cortical inhibition, increased reliance on spinal and brainstem pathways, or poor sensorimotor integration in the cortex. We cannot rule out Type II error as a factor in our results, and larger samples would be highly desirable for future studies. Additionally, this study tested the feasibility of minimizing muscle activity for passive pedaling. We found that a high proportion of both stroke and control subjects were unable to perform passive pedaling. This finding may reflect interindividual and sex differences in the ability to alter descending drive, inhibit spinal reflexes, or inhibit pattern generating circuits.

\section{Acknowledgments}

The authors would like to acknowledge Mary Asma for her assistance with data collection and analysis. This work was supported by American Heart Association-Midwest Affiliate (grant \#16PRE26650000; Cleland) and by the National Center for Medical Rehabilitation Research in the National Institute of Child Health and Human Development (grant \#HD060693; Schindler-Ivens).

\section{References}

Alary, F., Doyon, B., Loubinoux, I., Carel, C., Boulanouar, K., Ranjeva, J.P., . . . Chollet, F. (1998). Event-related potentials elicited by passive movements in humans: Characterization, source analysis, and comparison to fMRI. NeuroImage, 8(4), 377-390. doi: 10.1006/nimg.1998.0377

Ambrosini, E., Ferrante, S., Ferrigno, G., Molteni, F., \& Pedrocchi, A. (2012). Cycling induced by electrical stimulation improves muscle activation and symmetry during pedaling in hemiparetic patients. IEEE Transactions on Neural Systems and Rehabilitation Engineering, 20(3), 320-330. PubMed doi:10.1109/TNSRE.2012.2191574

Baron, J.C., Cohen, L.G., Cramer, S.C., Dobkin, B.H., Johansen-Berg, H., Loubinoux, I., . . . First International Workshop on Neuroimaging and Stroke Recovery. (2004). Neuroimaging in stroke recovery: A position paper from the first international workshop on neuroimaging and stroke recovery. Cerebrovascular Diseases, 18(3), 260-267. PubMed doi:10.1159/000080293

Biernaskie, J., \& Corbett, D. (2001). Enriched rehabilitative training promotes improved forelimb motor function and enhanced dendritic growth after focal ischemic injury. Journal of Neuroscience, 21(14), 5272-5280.

Blatow, M., Reinhardt, J., Riffel, K., Nennig, E., Wengenroth, M., \& Stippich, C. (2011). Clinical functional MRI of sensorimotor cortex using passive motor and sensory stimulation at 3 Tesla. Journal of Magnetic Resonance Imaging, 34(2), 429-437. PubMed doi:10.1002/jmri.22629

Boscolo Galazzo, I., Storti, S.F., Formaggio, E., Pizzini, F.B., Fiaschi, A., Beltramello, A., . . Manganotti, P. (2014). Investigation of brain hemodynamic changes induced by active and passive movements: A combined arterial spin labeling-BOLD fMRI study. 
Journal of Magnetic Resonance Imaging, 40(4), 937-948. PubMed doi:10.1002/ jmri.24432

Bosnell, R.A., Kincses, T., Stagg, C.J., Tomassini, V., Kischka, U., Jbabdi, S., . . JohansenBerg, H. (2011). Motor practice promotes increased activity in brain regions structurally disconnected after subcortical stroke. Neurorehabilitation \& Neural Repair, 25(7), 607-616. PubMed doi:10.1177/1545968311405675

Bowden, M.G., Balasubramanian, C.K., Neptune, R.R., \& Kautz, S.A. (2006). Anteriorposterior ground reaction forces as a measure of paretic leg contribution in hemiparetic walking. Stroke, 37(3), 872-876. PubMed doi:10.1161/01.STR.0000204063.75779.8d

Brooke, J.D., McIlroy, W.E., \& Collins, D.F. (1992). Movement features and H-reflex modulation. I. Pedalling versus matched controls. Brain Research, 582(1), 78-84. PubMed doi:10.1016/0006-8993(92)90319-5

Brooke, J.D., Misiaszek, J.E., \& Cheng, J. (1993). Locomotor-like rotation of either hip or knee inhibits soleus H reflexes in humans. Somatosensory \& Motor Research, 10(4), 357-364. PubMed doi:10.3109/08990229309028843

Brown, D.A., \& Kautz, S.A. (1998). Increased workload enhances force output during pedaling exercise in persons with poststroke hemiplegia. Stroke, 29(3), 598-606. PubMed doi:10.1161/01.STR.29.3.598

Bury, S.D., \& Jones, T.A. (2002). Unilateral sensorimotor cortex lesions in adult rats facilitate motor skill learning with the "unaffected" forelimb and training-induced dendritic structural plasticity in the motor cortex. Journal of Neuroscience, 22(19), 8597-8606. PubMed

Byrnes, M.L., Thickbroom, G.W., Phillips, B.A., Wilson, S.A., \& Mastaglia, F.L. (1999). Physiological studies of the corticomotor projection to the hand after subcortical stroke. Clinical Neurophysiology, 110(3), 487-498. PubMed doi:10.1016/S1388-2457(98)00044-3

Calautti, C., \& Baron, J.C. (2003). Functional neuroimaging studies of motor recovery after stroke in adults: A review. Stroke, 34(6), 1553-1566. PubMed doi:10.1161/01.STR. 0000071761.36075.A6

Calautti, C., Leroy, F., Guincestre, J.Y., \& Baron, J.C. (2001). Dynamics of motor network overactivation after striatocapsular stroke: A longitudinal PET study using a fixedperformance paradigm. Stroke, 32(11), 2534-2542. PubMed doi:10.1161/hs1101. 097401

Carmichael, S.T., Wei, L., Rovainen, C.M., \& Woolsey, T.A. (2001). New patterns of intracortical projections after focal cortical stroke. Neurobiology of Disease, 8(5), 910-922. PubMed doi:10.1006/nbdi.2001.0425

Christensen, L.O., Johannsen, P., Sinkjaer, T., Petersen, N., Pyndt, H.S., \& Nielsen, J.B. (2000). Cerebral activation during bicycle movements in man. Experimental Brain Research, 135(1), 66-72. PubMed doi:10.1007/s002210000493

Cirstea, M.C., \& Levin, M.F. (2000). Compensatory strategies for reaching in stroke. Brain, 123(Pt. 5), 940-953. doi:10.1093/brain/123.5.940

Cleland, B., \& Schindler-Ivens, S. (2015, October). Reduced pedaling related brain activation volume post-stroke does not depend on task performance. Presented at the Society for Neuroscience National Meeting, Chicago, IL.

Coxon, J.P., Stinear, C.M., \& Byblow, W.D. (2006). Intracortical inhibition during volitional inhibition of prepared action. Journal of Neurophysiology, 95(6), 3371-3383. PubMed doi:10.1152/jn.01334.2005

Cramer, S.C., Nelles, G., Benson, R.R., Kaplan, J.D., Parker, R.A., Kwong, K.K., . . Rosen, B.R. (1997). A functional MRI study of subjects recovered from hemiparetic stroke. Stroke, 28(12), 2518-2527. PubMed doi:10.1161/01.STR.28.12.2518

Cruz, E.G., Waldinger, H.C., \& Kamper, D.G. (2005). Kinetic and kinematic workspaces of the index finger following stroke. Brain, 128(Pt. 5), 1112-1121. PubMed doi:10.1093/ brain/awh432 
Dancause, N., Barbay, S., Frost, S.B., Plautz, E.J., Chen, D., Zoubina, E.V., . . Nudo, R.J. (2005). Extensive cortical rewiring after brain injury. Journal of Neuroscience, 25(44), 10167-10179. PubMed doi:10.1523/JNEUROSCI.3256-05.2005

DeYoe, E.A., Bandettini, P., Neitz, J., Miller, D., \& Winans, P. (1994). Functional magnetic resonance imaging (FMRI) of the human brain. Journal of Neuroscience Methods, 54(2), 171-187. PubMed doi:10.1016/0165-0270(94)90191-0

Dobkin, B.H., Firestine, A., West, M., Saremi, K., \& Woods, R. (2004). Ankle dorsiflexion as an fMRI paradigm to assay motor control for walking during rehabilitation. NeuroImage, 23(1), 370-381. doi:10.1016/j.neuroimage.2004.06.008

Enzinger, C., Johansen-Berg, H., Dawes, H., Bogdanovic, M., Collett, J., Guy, C., . . . Matthews, P.M. (2008). Functional MRI correlates of lower limb function in stroke victims with gait impairment. Stroke, 39(5), 1507-1513. PubMed doi:10.1161/ STROKEAHA.107.501999

Feys, H., Van Hees, J., Bruyninckx, F., Mercelis, R., \& De Weerdt, W. (2000). Value of somatosensory and motor evoked potentials in predicting arm recovery after a stroke. Journal of Neurology, Neurosurgery, \& Psychiatry, 68(3), 323-331. PubMed doi: 10.1136/jnnp.68.3.323

Friedman, D.B., Friberg, L., Mitchell, J.H., \& Secher, N.H. (1991). Effect of axillary blockade on regional cerebral blood flow during static handgrip. Journal of Applied Physiology, 71(2), 651-656. PubMed doi:10.1152/jappl.1991.71.2.651

Friedman, D.B., Friberg, L., Payne, G., Mitchell, J.H., \& Secher, N.H. (1992). Effects of axillary blockade on regional cerebral blood flow during dynamic hand contractions. Journal of Applied Physiology, 73(5), 2120-2125. PubMed doi:10.1152/jappl.1992. 73.5.2120

Fuchs, D.P., Sanghvi, N., Wieser, J., \& Schindler-Ivens, S. (2011). Pedaling alters the excitability and modulation of vastus medialis H-reflexes after stroke. Clinical Neurophysiology, 122(10), 2036-2043. PubMed doi:10.1016/j.clinph.2011.03.010

Grefkes, C., Eickhoff, S.B., Nowak, D.A., Dafotakis, M., \& Fink, G.R. (2008). Dynamic intra- and interhemispheric interactions during unilateral and bilateral hand movements assessed with fMRI and DCM. NeuroImage, 41(4), 1382-1394. doi:10.1016/ j.neuroimage.2008.03.048

Grey, M.J., Nielsen, J.B., Mazzaro, N., \& Sinkjaer, T. (2007). Positive force feedback in human walking. Journal of Neurophysiology, 581(Pt. 1), 99-105. doi:10.1113/jphysiol. 2007.130088

Guzzetta, A., Staudt, M., Petacchi, E., Ehlers, J., Erb, M., Wilke, M., . . Cioni, G. (2007). Brain representation of active and passive hand movements in children. Pediatric Research, 61(4), 485-490. PubMed doi:10.1203/pdr.0b013e3180332c2e

Hamzei, F., Dettmers, C., Rijntjes, M., \& Weiller, C. (2008). The effect of cortico-spinal tract damage on primary sensorimotor cortex activation after rehabilitation therapy. Experimental Brain Research, 190(3), 329-336. PubMed doi:10.1007/s00221-008-1474-x

Jain, S., Gourab, K., Schindler-Ivens, S., \& Schmit, B.D. (2013). EEG during pedaling: Evidence for cortical control of locomotor tasks. Clinical Neurophysiology, 124(2), 379-390. PubMed doi:10.1016/j.clinph.2012.08.021

Kalinosky, B.T., Schindler-Ivens, S., \& Schmit, B.D. (2013). White matter structural connectivity is associated with sensorimotor function in stroke survivors. NeuroImage: Clinical, 2, 767-781. doi:10.1016/j.nicl.2013.05.009

Kautz, S.A., Patten, C., \& Neptune, R.R. (2006). Does unilateral pedaling activate a rhythmic locomotor pattern in the nonpedaling leg in post-stroke hemiparesis? Journal of Neurophysiology, 95(5), 3154-3163. PubMed doi:10.1152/jn.00951.2005

Kim, Y.H., You, S.H., Kwon, Y.H., Hallett, M., Kim, J.H., \& Jang, S.H. (2006). Longitudinal fMRI study for locomotor recovery in patients with stroke. Neurology, 67(2), 330-333. PubMed doi:10.1212/01.wnl.0000225178.85833.0d 
Knutsson, E., \& Richards, C. (1979). Different types of disturbed motor control in gait of hemiparetic patients. Brain, 102(2), 405-430. PubMed doi:10.1093/brain/102. 2.405

Kocak, M., Ulmer, J.L., Sahin Ugurel, M., Gaggl, W., \& Prost, R.W. (2009). Motor homunculus: Passive mapping in healthy volunteers by using functional MR imaging-Initial results. Radiology, 251(2), 485-492. PubMed doi:10.1148/radiol.2512080231

Krakauer, J.W. (2007). Avoiding performance and task confounds: Multimodal investigation of brain reorganization after stroke rehabilitation. Experimental Neurology, 204(2), 491-495. PubMed doi:10.1016/j.expneurol.2006.12.026

La Joie, W.J., Reddy, N.M., \& Melvin, J.L. (1982). Somatosensory evoked potentials: Their predictive value in right hemiplegia. Archives of Physical Medicine and Rehabilitation, 63(5), 223-226. PubMed

Larsen, B., Voight, M., \& Grey, M.J. (2006). Changes in the soleus stretch reflex at different pedaling frequencies and crank loads during pedaling. Motor Control, 10(3), 265-279. PubMed doi:10.1123/mcj.10.3.265

Levin, M.F., Kleim, J.A., \& Wolf, S.L. (2009). What do motor "recovery" and "compensation" mean in patients following stroke? Neurorehabilitation \& Neural Repair, 23(4), 313-319. PubMed doi:10.1177/1545968308328727

Liepert, J., Uhde, I., Gräf, S., Leidner, O., \& Weiller, C. (2001). Motor cortex plasticity during forced-use therapy in stroke patients: A preliminary study. Journal of Neurology, 248(4), 315-321. PubMed doi:10.1007/s004150170207

Logothetis, N.K. (2008). What we can do and what we cannot do with fMRI. Nature, 453(7197), 869-878. PubMed doi:10.1038/nature06976

McIlroy, W.E., Collins, D.F., \& Brooke, J.D. (1992). Movement features and H-reflex modulation. II. Passive rotation, movement velocity and single leg movement. Brain Research, 582(1), 85-93. PubMed doi:10.1016/0006-8993(92)90320-9

Mehta, J.P., Verber, M.D., Wieser, J.A., Schmit, B.D., \& Schindler-Ivens, S.M. (2009). A novel technique for examining human brain activity associated with pedaling using fMRI. Journal of Neuroscience Methods, 179(2), 230-239. PubMed doi:10.1016/ j.jneumeth.2009.01.029

Mehta, J.P., Verber, M.D., Wieser, J.A., Schmit, B.D., \& Schindler-Ivens, S.M. (2012). The effect of movement rate and complexity on functional magnetic resonance signal change during pedaling. Motor Control, 16(2), 158-175. PubMed doi:10.1123/mcj. 16.2.158

Miyai, I., Yagura, H., Hatakenaka, M., Oda, I., Konishi, I., \& Kubota, K. (2003). Longitudinal optical imaging study for locomotor recovery after stroke. Stroke, 34(12), 2866-2870. PubMed doi:10.1161/01.STR.0000100166.81077.8A

Miyai, I., Yagura, H., Oda, I., Konishi, I., Eda, H., Suzuki, T., \& Kubota, K. (2002). Premotor cortex is involved in restoration of gait in stroke. Annals of Neurology, 52(2), 188-194. PubMed doi:10.1002/ana.10274

Motawar, B., Hur, P., Stinear, J., \& Seo, N.J. (2012). Contribution of intracortical inhibition in voluntary muscle relaxation. Experimental Brain Research, 221(3), 299-308. PubMed doi:10.1007/s00221-012-3173-x

Motl, R.W., Knowles, B.D., \& Dishman, R.K. (2003). Acute bouts of active and passive leg cycling attenuate the amplitude of the soleus H-reflex in humans. Neuroscience Letters, 347(2), 69-72. PubMed doi:10.1016/S0304-3940(03)00652-9

Murase, N., Duque, J., Mazzocchio, R., \& Cohen, L.G. (2004). Influence of interhemispheric interactions on motor function in chronic stroke. Annals of Neurology, 55(3), 400-409. PubMed doi:10.1002/ana.10848

Nielsen, J., Crone, C., \& Hultborn, H. (1993). H-reflexes are smaller in dancers from the Royal Danish Ballet than in well-trained athletes. European Journal of Applied Physiology, 66(2), 116-121. doi:10.1007/BF01427051 
Noble, J.W., Eng, J.J., \& Boyd, L.A. (2014). Bilateral motor tasks involve more brain regions and higher neural activation than unilateral tasks: An fMRI study. Experimental Brain Research, 232(9), 2785-2795. PubMed doi:10.1007/s00221-014-3963-4

Onishi, H., Sugawara, K., Yamashiro, K., Sato, D., Suzuki, M., Kirimoto, H., . . . Kameyama, S. (2013). Neuromagnetic activation following active and passive finger movements. Brain and Behavior, 3(2), 178-192. PubMed doi:10.1002/brb3.126

Perell, K.L., Gregor, R.J., \& Scremin, A.M.E. (1998). Lower limb cycling mechanics in subjects with unilateral cerebrovascular accidents. Journal of Applied Biomechanics, 14, 158-179. doi:10.1123/jab.14.2.158

Power, M.L., \& Schulkin, J. (2008). Sex differences in fat storage, fat metabolism, and the health risks from obesity: Possible evolutionary origins. British Journal of Nutrition, 99(5), 931-940. PubMed doi:10.1017/S0007114507853347

Prochazka, A., \& Ellaway, P. (2012). Sensory systems in the control of movement. Comprehensive Physiology, 2(4), 2615-2627. PubMed doi:10.1002/cphy.c100086

Promjunyakul, N.O., Schmit, B.D., \& Schindler-Ivens, S.M. (2015). A novel fMRI paradigm suggests that pedaling-related brain activation is altered after stroke. Frontiers in Human Neuroscience, 9, 324. PubMed doi:10.3389/fnhum.2015.00324

Qü, M., Buchkremer-Ratzmann, I., Schiene, K., Schroeter, M., Witte, O.W., \& Zilles, K. (1998). Bihemispheric reduction of GABAA receptor binding following focal cortical photothrombotic lesions in the rat brain. Brain Research, 813(2), 374-380. doi:10. 1016/S0006-8993(98)01063-4

Reddy, H., Floyer, A., Donaghy, M., \& Matthews, P.M. (2001). Altered cortical activation with finger movement after peripheral denervation: Comparison of active and passive tasks. Experimental Brain Research, 138(4), 484-491. PubMed doi:10.1007/s002210100732

Schiene, K., Bruehl, C., Zilles, K., Qü, M., Hagemann, G., Kraemer, M., \& Witte, O.W. (1996). Neuronal hyperexcitability and reduction of GABAA-receptor expression in the surround of cerebral photothrombosis. Journal of Cerebral Blood Flow \& Metabolism, 16(5), 906-914. PubMed doi:10.1097/00004647-199609000-00014

Schindler-Ivens, S., Brown, D.A., Lewis, G.N., Nielsen, J.B., Ondishko, K.L., \& Wieser, J. (2008). Soleus H-reflex excitability during pedaling post-stroke. Experimental Brain Research, 188(3), 465-474. PubMed doi:10.1007/s00221-008-1373-1

Schmahmann, J.D., Doyon, J., McDonald, D., Holmes, C., Lavoie, K., Hurwitz, A.S., . . Petrides, M. (1999). Three-dimensional MRI atlas of the human cerebellum in proportional stereotaxic space. NeuroImage, 10(3 Pt. 1), 233-260. doi:10.1006/ nimg.1999.0459

Sinkjaer, T., Andersen, J.B., Ladouceur, M., Christensen, L.O., \& Nielsen, J.B. (2000). Major role for sensory feedback in soleus EMG activity in the stance phase of walking in man. Journal of Neurophysiology, 523(Pt. 3), 817-827.

Stephens, M.J., \& Yang, J.F. (1999). Loading during the stance phase of walking in humans increases the extensor EMG amplitude but does not change the duration of the step cycle. Experimental Brain Research, 124(3), 363-370. PubMed doi:10.1007/ s002210050633

Takemi, M., Masakado, Y., Liu, M., \& Ushiba, J. (2013). Event-related desynchronization reflects downregulation of intracortical inhibition in human primary motor cortex. Journal of Neurophysiology, 110(5), 1158-1166. PubMed doi:10.1152/jn.01092.2012

Terumitsu, M., Ikeda, K., Kwee, I.L., \& Nakada, T. (2009). Participation of primary motor cortex area 4a in complex sensory processing: 3.0-T fMRI study. NeuroReport, 20(7), 679-683. doi:10.1097/WNR.0b013e32832a1820

Toma, K., Honda, M., Hanakawa, T., Okada, T., Fukuyama, H., Ikeda, A., . . Shibasaki, H. (1999). Activities of the primary and supplementary motor areas increase in preparation and execution of voluntary muscle relaxation: An event-related fMRI study. Journal of Neuroscience, 19(9), 3527-3534. PubMed 
Traversa, R., Cicinelli, P., Pasqualetti, P., Filippi, M., \& Rossini, P.M. (1998). Follow-up of interhemispheric differences of motor evoked potentials from the 'affected' and 'unaffected' hemispheres in human stroke. Brain Research, 803(1-2), 1-8. PubMed doi:10.1016/S0006-8993(98)00505-8

Verschueren, S.M., Swinnen, S.P., Desloovere, K., \& Duysens, J. (2002). Effects of tendon vibration on the spatiotemporal characteristics of human locomotion. Experimental Brain Research, 143(2), 231-239. PubMed doi:10.1007/s00221-001-0987-3

Vlaar, M.P., Solis-Escalante, T., Dewald, J.P.A., van Wegen, E.E.H., Schouten, A.C., Kwakkel, G., . . . 4D-EEG consortium. (2017). Quantification of task-dependent cortical activation evoked by robotic continuous wrist joint manipulation in chronic hemiparetic stroke. Journal of NeuroEngineering and Rehabilitation, 14(1), 30. PubMed doi:10.1186/s12984-017-0240-3

Ward, N.S., Brown, M.M., Thompson, A.J., \& Frackowiak, R.S. (2003a). Neural correlates of motor recovery after stroke: A longitudinal fMRI study. Brain, 126(Pt. 11), 2476-2496. doi:10.1093/brain/awg245

Ward, N.S., Brown, M.M., Thompson, A.J., \& Frackowiak, R.S. (2003b). Neural correlates of outcome after stroke: A cross-sectional fMRI study. Brain, 126(Pt. 6), 1430-1448. doi:10.1093/brain/awg145

Weiller, C., Chollet, F., Friston, K.J., Wise, R.J., \& Frackowiak, R.S. (1992). Functional reorganization of the brain in recovery from striatocapsular infarction in man. Annals of Neurology, 31(5), 463-472. PubMed doi:10.1002/ana.410310502

Weiller, C., Jüptner, M., Fellows, S., Rijntjes, M., Leonhardt, G., Kiebel, S., . . Thilmann, A.F. (1996). Brain representation of active and passive movements. NeuroImage, 4(2), 105-110. doi:10.1006/nimg.1996.0034

Wexler, B.E., Fulbright, R.K., Lacadie, C.M., Skudlarski, P., Kelz, M.B., Constable, R.T., \& Gore, J.C. (1997). An fMRI study of the human cortical motor system response to increasing functional demands. Magnetic Resonance Imaging, 15(4), 385-396. PubMed doi:10.1016/S0730-725X(96)00232-9

Yamaguchi, T., Fujiwara, T., Liu, W., \& Liu, M. (2012). Effects of pedaling exercise on the intracortical inhibition of cortical leg area. Experimental Brain Research, 218(3), 401-406. PubMed doi:10.1007/s00221-012-3026-7

Yang, J.F., Stein, R.B., \& James, K.B. (1991). Contribution of peripheral afferents to the activation of the soleus muscle during walking in humans. Experimental Brain Research, 87(3), 679-687. PubMed doi:10.1007/BF00227094

Zhang, Y., Li, K.S., Ning, Y.Z., Fu, C.H., Liu, H.W., Han, X., . . Zou, Y.H. (2016). Altered structural and functional connectivity between the bilateral primary motor cortex in unilateral subcortical stroke: A multimodal magnetic resonance imaging study. Medicine (Baltimore), 95(31), e4534. doi:10.1097/MD.0000000000004534 